\title{
Superficial Temporal Artery - Middle Cerebral Artery Bypass in Moyamoya Disease Treatment
}

\author{
Aleksejs Repnikovs*, Kaspars Auslands**, Sergejs Vedenskijs**, Rolfs Ozols** \\ * Riga Stradns University, Faculty of Medicine, Riga, Latvia \\ **Riga Eastern Clinical University Hospital Gailezers, Department of Neurosurgery, Riga, Latvia
}

\section{Summary}

Moyamoya disease is the chronic cerebrovascular disease, which is characterized by progressive occlusion of the intracranial part of internal carotid arteries and their proximal branches. Revascularization surgery of the ischemic hemisphere is a frequently recommended kind of treatment Moyamoya disease patients and it allows to prevent against stroke and to improve blood perfusion of the brain. We present the one case with treatment of this disease using superficial temporal artery (STA) - middle cerebral artery (MCA) bypass.

Key words: Moyamoya disease, STA-MCA bypass

\section{AIM OF THE DEMONSTRATION}

The aim of this demonstration is to report about our experience in treatment of rare in our region patient with Moyamoya disease.

\section{CASE REPORT}

55-year old woman was presented with history of ischemic strokes in the left anterior cerebral artery perfusion area 4 years ago and in the right middle cerebral artery perfusion area 2 years ago. Headaches, vomiting, dizziness, seizures, transient ischemic attacks, which are characterized as weakness or sensory loss during few minutes, speech difficulty, darkening of vision during few seconds, were presented in a current of 5 years and these neurological symptoms became more frequent and worse.

Preoperative MRI examination of the brain showed the old cerebral infarcts in the left frontal and right parietotemporal region.

Preoperative A right and left carotid angiograms (Fig.1) were performed with defuse stenosis of both side internal carotid arteries and the classical "puff of smoke" appearance was seen in the right and left cerebral circulation (Fig.2).

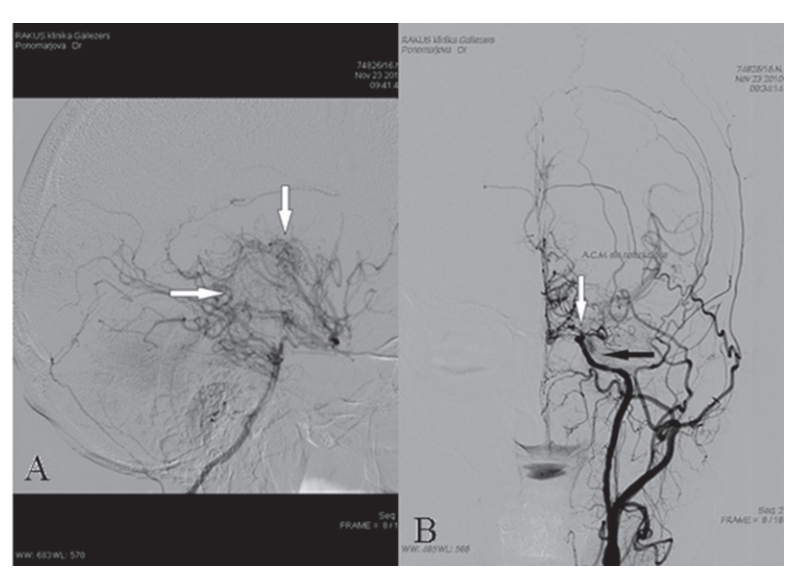

Fig. 1 - Angiogram A, lateral projection, shows abnormal collateral vessels network (white arrows) and the angiogram $B$ shows defuse stenosis of the internal carotid artery (black arrow) with occlusion of ophtalmica segment (white arrow).

The first operation was done on the right hemisphere on $5^{\text {th }}$ of January 2011 and the second - on the left hemisphere on $2^{\text {nd }}$ of May 2011. In this surgical procedures were performed both side STA-MCA bypass, where STA was used as a donor artery and MCA as a recipient artery.

Few days after each operation patient felt much better and noted that arms and legs became agile and more powerful. During the year coming to examinations and controls patient had not complained of symptoms, which were presented before, except headaches.

Postoperative angiograms were performed, that, during the year, anastomoses work on both sides and were noticeably reduced quantity of Moyamoya vessels (Fig.3) 




Fig. 2 - Angiogram A, lateral projection, shows reduced quantity of abnormal collateral vessels (white arrows). Angiogram B shows excellent filling of the MCA (white arrow) from the STAMCA bypass (black arrow).

\section{DISCUSSION}

The etiology of Moyamoya disease is unknown, but the pathological analysis had revealed, that this disease is characterized by the intimal thickening and attenuation of tunica media of the proximal vessels of the circle of Willis as well as the development of small collateral net-like vessels. The primary proteins that are currently implicated in the pathophysiology of Moyamoya disease include vascular endothelial growth factor, basic fibroblast growth factor, hepatocyte growth factor, transforming growth factor- $\beta_{1}$, and granulocyte colonystimulating factor. Also the current literature has pointed to a low penetrance autosomal dominant or polygenic mode of transmittance at loci on chromosomes $3,6,8$, 12 , and 17 (13), as well as specific alleles of class II genes of the human leukocyte antigen have been described. (5)

Nowadays therapy is aimed on preventive actions against a stroke and to improve circulation of the brain, which helps prevent the appearance of symptoms and complications, which is characterized by this disease.

The surgery treatment is directed on creation of new blood supply for brain region with poor blood perfusion. There exist two main methods of surgical revascularization: direct and indirect methods.

Direct method includes the creation of anastomosis between branches of external carotid artery and internal carotid artery, and this method is typically used in treatment for adult patients. In general is used the superficial temporal artery to middle cerebral artery bypass. (4) This method the first time was performed by Yasargil in 1972 (2) and it allows immediately increase perfusion of the ischemic brain region.

Indirect techniques, which are traditionally used for children, involve the placement of vascularized tissues, which are supplied by the external carotid artery, in direct contact with the ischaemic hemisphere surface, leading to germinate new blood vessels to the underlying cerebral cortex. (12) There are few methods of indirect revascularization: the superficial temporal artery, which is left in continuity, or the temporal muscle on the ischaemic side of the head is placed in direct contact with the surface of the brain, as well as multiple burr holes procedure, in which several small holes are placed in the skull. Over time the result of angiogenesis is the formation of small arterial vessels, which germinate into the brain and increase the blood perfusion.(7) (8) (9) (10) There are possible negative effects of this method: necessary time for neovascularization, that means, that we cannot evaluate effect in short time after surgery, because of new vessels need at least few months to germinate into the brain and there is a possibility that process of angiogenesis could be not enough to provide normal perfusion of the brain.(3)

We decided to use STA-MCA bypass in treatment of our Moyamoya patient, because this is the most studied method of management Moyamoya disease, and this method allow immediately realise our main aim - increase blood flow in ischemic hemispheres. Also there is a possibility to use a combination of both revascularization methods with good short-term and long-term outcomes. This treatment could be used as a first step in Moyamoya disease treatment or after unsuccessful indirect surgery, when neovascularization process cannot provide necessary blood perfusion. (1) (4) (6) (11)

\section{Conflict of interest: None}

\section{REFERENCES}

1. Czabanka M, Peña-Tapia P, Scharf J, Schubert GA, Münch E, Horn P, Schmiedek P, Vajkoczy P Characterization of direct and indirect cerebral revascularization for the treatment of European patients with moyamoya disease. //Cerebrovasc Dis. $2011 ; 32(4): 361-9$.

2. Donaghy RM: Neurologic surgery. //Surg Gynecol Obstet 1972; 134: 269-270.

3. Fung LW, Thompson D, Ganesan V. Revascularisation surgery for paediatric moyamoya: a review of the literature. //Childs Nerv Syst 2005; 21:358-64.

4. Golby AJ, Marks MP, Thompson RC, Steinberg GK. Direct and combined revascularization in pediatric moyamoya disease. //Neurosurgery. 1999; 45:50-58.

5. Han H, Pyo CW, Yoo DS, Huh PW, Cho KS, Kim DS. Associations of Moyamoya patients with HLA class I and class II alleles in the Korean population. // J Korean Med Sci 2003; 18:876-80.

6. Ikezaki K. Rational approach to treatment of moyamoya disease in childhood. / J Child Neurol 2000; 15:350-6.

7. Karasawa J, Touho H, Ohnishi H, Miyamoto S, Kikuchi H: Cerebral revascularization using omental transplantation for childhood moyamoya disease. // J Neurosurg 1993; 79:192-196.

8. Karasawa J, Kikuchi H, Furuse S, Sakaki T, Yoshida Y: A surgical treatment of "moyamoya" disease "encephalo-myo synangiosis". //Neurol Med Chir (Tokyo) 1977; 17:29-37. 
9. Kawaguchi T, Fujita S, Hosoda K, Shose Y, Hamano S, Iwakura M, et al: Multiple burr-hole operation for adult moyamoya disease.// J Neurosurg 1996; 84:468-476.

10. Kinugasa K, Mandai S, Kamata I, Sugiu K, Ohmoto T: Surgical treatment of moyamoya disease: Operative technique for encephalo-duro-arterio-myosynangiosis, its follow-up, clinical results, and angiograms. //Neurosurgery 1993; 32:527-531.

11. Matsushima $\mathrm{T}$, Inoue $\mathrm{T}$, Ikezaki $\mathrm{K}$, et al. Multiple combined indirect procedure for the surgical treatment of children with moyamoya disease: a comparison with single indirect anastomosis with direct anastomosis. //Neurosurg Focus 1998; 5(5):e4.

12. Matsushima $\mathrm{T}$, Inoue $\mathrm{T}$, Katsuta $\mathrm{T}$, et al. An indirect revascularization method in the surgical treatment of moyamoya disease - various kinds of indirect procedures and a multiple combined indirect procedure. //Neurol Med Chir (Tokyo) 1998; 38:Suppl:297-302.

13. Weinberg DG, Arnaout OM, Rahme RJ, Aoun SG, Batjer HH, Bendok BR. Moyamoya disease: a review of histopathology, biochemistry, and genetics. // Neurosurg Focus. 2011 Jun; 30(6):E20.

\section{Address:}

Aleksejs Repnikovs,

Kandavas street 23-32

LV-5400, Daugavpils, Latvia

E-mail: arepnikov@inbox.lv 